\title{
Errata: "Statistical Characteristics of Inhomogeneities of Dislocation Networks" Published in Crystallography Reports, 2002, vol. 47, no. 3, pp. 475-479.
}

\section{E. I. Gutorov}

1. Abstract: the third line should read "by the functions of the type $f_{k}(x)=n(n x)^{n-1} e^{-n x} /(n-1) \ldots$...

2. The heading of column 12 of Table 2 should read CdS(19).

3. Line 2 in the right-hand column on page 477 should read "...and in zinc [17] ...".

4. Expressions (2) and (3) should read

$$
F(x)=\frac{5^{5}}{4 !} x^{4} e^{-5 x}
$$

and

$$
F_{n}(x)=\frac{n(n x)^{n-1}}{(n-1) !} e^{-n x}
$$

respectively. 